\title{
Bienestar psicológico y estrés laboral en trabajadores no profesionales de la ciudad de Ambato, aplicando el cuestionario de salud general de Goldberg GHQ-12
}

Psychological well-being and work stress in non-professional workers in the city of Ambato, applying the Goldberg GHQ-12 general health questionnaire

Luis Fernando Taruchaín Pozo. ${ }^{1}$, Verónica Adriana Freire Palacios. ${ }^{2}$ \& María de los Ángeles

$$
\text { Mayorga Álvarez. }{ }^{3}
$$

\section{Recibido: 15-01-2020 / Revisado: 23-01-2020 /Aceptado: 26-01-2020/ Publicado: 27-02-2020}

\section{Abstract \\ DOI: https://doi.org/10.33262/cienciadigital.v4i1.1.1162}

Objective. Determine psychological well-being and work stress in non-professional workers in the city of Ambato, applying the Goldberg GHQ-12 general health questionnaire.

Methodology. Descriptive cross-sectional study, where the Goldberg General Health Questionnaire (GHQ-12) was used to prove to be an effective tool for the assessment of mental health in the general population and in the clinical population, being one of the most validated screening instruments used worldwide and one of the most recommended to be used in health surveys. The analysis of internal consistency using Cronbach's alpha coefficient showed good internal consistency and validity (.84). The sample consisted of 280 non-professional workers from different marketing centers located in the city of Ambato. Results Of the 280 participants studied, $40 \%$ were men and $60 \%$ women, with a level of work stress between very high and high at $61 \%$, and, within what is self-perceived psychological well-being, most Rate it as very low and low at $59 \%$. This allows us to conclude that stress affects all types of people regardless of age, gender, marital status, socio-economic status and level of education, since all are affected by external and internal environments that are difficult to manage.

\footnotetext{
1 Universidad Tecnológica Indoamérica, Facultad de Ciencias Humanas y de la Salud, Ambato, Ecuador, Psicólogo Industrial, fernandotaruchain@uti.edu.ec,_ORCID: http://orcid.org/0000-0002$1315-5955$

${ }^{2}$ Universidad Técnica de Ambato, Facultad de Ciencias Humanas y de la Educación, Carrera de Educación Básica, Psicóloga Clínica, va.freire@uta.edu.ec, ORCID: http://orcid.org/0000-0001-9493-6552

3 Empresa de Capacitación, Asesoría e Investigación "EMCASIN", Ambato, Ecuador, Gerente Investigador, mayorga maria@yahoo.es, ORCID: https://orcid.org/0000-0001-7419-4520
} 
Keywords: Work stress, mental health, psychological well-being, GHQ-12

\section{Resumen}

Objetivo. Determinar el bienestar psicológico y estrés laboral en trabajadores no profesionales de la ciudad de Ambato, aplicando el cuestionario de salud general de Goldberg GHQ-12.

Metodología. Estudio descriptivo con corte transversal, en donde se utilizó el Cuestionario de Salud General Goldberg (GHQ-12) demostrado ser una herramienta efectiva para la valoración de la salud mental en población general y en población clínica, siendo uno de los instrumentos de tamizaje validado más utilizado en todo el mundo y uno de los más recomendados para ser empleado en encuestas de salud. El análisis de consistencia interna utilizando el coeficiente alpha de Cronbach, mostró una buena consistencia interna y validez (.84). La muestra estuvo constituida por 280 trabajadores no profesionales de distintos centros de comercialización ubicados en la ciudad de Ambato. Resultados. De los 280 participantes estudiados, el $40 \%$ eran hombres y el $60 \%$ mujeres, con un nivel de estrés laboral entre muy alto y alto en un $61 \%$, y, dentro de lo que es el bienestar psicológico auto percibido, la mayor parte lo valora como muy bajo y bajo en un 59\%. Lo que permite concluir que el estrés afecta a todo tipo de personas sin considerar, edad, género, estado civil, condición socio-económica y nivel de escolaridad, pues todos se afectados por entornos externos e internos difíciles de manejar

Palabras clave: Estrés laboral, salud mental, bienestar psicológico, GHQ-12

\section{INTRODUCCIÓN}

Para la Organización Mundial de la Salud (OMS), la salud mental corresponde: "un estado de completo bienestar físico, mental y social, y no solamente la ausencia de afecciones o enfermedades" (Yegler, Diez, Gómez, \& Miralles, 2013). Otros autores definen el bienestar psicosocial incluye ausencia de enfermedad como indicador de desarrollo, e incluye la satisfacción de necesidades y el desarrollo de las potencialidades del ser humano, respetando su equidad y entorno (Arón, 2017).

En base a las premisas anteriormente expuestas y al analizar la profunda trasformación del mundo laboral, la inserción de nuevas tecnologías, el consumismo, la globalización, cambios organizacionales y gerenciales, políticas laborales, precarización del trabajo y, aumento del desempleo; así como también nuevos procesos de trabajo, se puede concluir que es lo que ha dado lugar a implicaciones subjetivas en el ser humano, incidiendo en el aparecimiento de factores de riesgo sicosociales que influyen directamente en la calidad de vida y el bienestar de los trabajadores, dando lugar a lo que se conoce como estrés laboral, siendo en la actualidad un fenómeno de interés general en la sociedad moderna (Díaz y Gómez, 2016). 
Mientras que para Gil-Monte (2016), el estrés en el trabajo, constituye:

Una respuesta al estrés laboral crónico, este aparece con frecuencia en profesionales del sector de servicios, que tienen interrelación directa con personas, destacándose por la aparición de cogniciones, de emociones, y de actitudes negativas hacia el trabajo, hacia las personas con las que se relaciona el individuo en su trabajo, en especial los clientes, y hacia el propio rol profesional. Como consecuencia de esta respuesta aparecen una serie de disfunciones conductuales, psicológicas, y fisiológicas, que van a tener repercusiones nocivas para las personas y para la organización (p. 5-14).

El Estrés es un proceso continuo, que va surgiendo de forma paulatina y que se va instaurando en el individuo hasta provocar en él la sintomatología específica (Ávila, 2014), se plantea que el foco del fenómeno está en el proceso de erosión psicológica y los resultados psicológicos y sociales de una exposición crónica a factores de ansiedad, esto se debe por la exposición prolongada a los estresores interpersonales crónicos en el trabajo y tiende a ser bastante estable con el tiempo. Asimismo, el estrés laboral no es una dificultad de las personas, sino el resultado del ambiente social en el cual trabaja, la estructura y el funcionamiento del lugar el que delimita la forma en la cual las personas interactúan y realizan sus trabajos (Díaz y Gómez, 2016); cuando se presentan unas condiciones laborales en las cuales no se reconoce el lado humano del trabajo, se genera una brecha muy amplia entre la naturaleza del trabajo y la de las personas, lo cual eleva el riesgo de desarrollo de estrés.

El estrés es un fenómeno que a menudo se presenta en la mayoría de los seres humanos, pero una vez que este empieza a transformarse en burnout, acaba siendo mucho más problemático, por lo que es imprescindible su identificación oportuna para combatir sus síntomas lo más pronto posible. para Aguirre y Gallo (2018), manifiestan que los periodos repetitivos de estrés suelen preceder al síndrome de burnout, por lo tanto, un burnout no se puede activar sino se experimentó previamente cierto grado de estrés (pp. 65-72). Existen determinadas actividades laborales en donde, los profesionales son más susceptibles a presentar estrés crónico o burnout. Sin embargo, se debe reconocer que estos signos o síntomas pueden presentarse en cualquier persona cuya actividad implique una relación constante con sus congéneres (Velázquez y Saldívar, 2018). Así se tienen investigaciones realizadas en otros países como la realizada por Pera (2016), realizada en España, en la cual determina que este tipo de profesionales que se dedican al comercio vivencian momentos muy diferentes a un trabajador formal que se encuentra en un trabajo con una remuneración fija y bajo condiciones previamente establecidas, los comerciantes se ven expuestos a una competencia feroz entre 
cadenas con márgenes de venta cada vez más estrechos; además este tipo de personas se ven expuestos a factores externos que lo hacen cada vez más vulnerable emocionalmente como son: jornadas semanales de 6 a 7 días con horarios repartidos de mañana, tarde y noche, fuentes de tensión inesperadas, nivel de auto-responsabilidad y auto exigencia muy alto, lo que les lleva a vivir desde el eustrés hasta el estrés positivo.

Otra investigación es la realizada por Barrera (2017), en la cual se menciona que las causas de estrés laboral en propietarios del comercio informal, encontrándose que: se logró Identificar como los principales desencadenantes de estrés laboral en los propietarios del comercio informal las bajas ventas, deudas, condiciones inadecuadas para realizar su trabajo, falta de conocimiento sobre el estrés laboral, descuido en la salud y problemas familiares. Se logró establecer que las consecuencias que surgen del estrés laboral en el entorno familiar, es la incapacidad de separar los problemas laborales y trasladarlos al ámbito familiar. Los propietarios del comercio informal no cuentan con las herramientas y la información necesaria para enfrentar el estrés laboral.

Es importante mencionar que para el caso de esta investigación el término no profesional se ha utilizado para hacer mención a personas que laboran en áreas del comercio y que por diferentes razones no han adquirido un título profesional. La ciudad de Ambato se caracteriza por tener una alta población que se dedica al comercio siendo una de las principales fuentes de ingreso económico para esta, por esta razón se consideró de suma importancia realizar este estudio en este tipo de personas que están expuestas a diferentes factores como: demanda excesiva tanto física como mental, horarios rotativos, sobrecarga trabajo, inestabilidad laboral, demanda excesiva tanto física como mental, horarios rotativos, sobrecarga trabajo, inestabilidad laboral, trabajo bajo presión, preocupaciones diversas, angustias, temores entre otros trabajo bajo presión, preocupaciones diversas, angustias, temores entre otros. estos factores hacen que este tipo de personas reflejan una interacción de exigencias, tensiones y nulo apoyo, dando como resultado un nivel deficiente de tolerancia al estrés, es decir, que el nivel de presión y actividades que realizan este tipo de trabajadores sobrepasa su capacidad.

Bajo este contexto el objetivo de esta investigación se centra en: Determinar el bienestar psicológico y estrés laboral en trabajadores no profesionales de la ciudad de Ambato, aplicando el cuestionario de salud general de Goldberg GHQ-12.

\section{METODOLOGÍA}

Diseño. El nivel de investigación es de tipo descriptivo, porque permite la descripción del bienestar psicológico y estrés laboral en trabajadores no profesionales de los centros 
comerciales de la ciudad de Ambato, esta descripción se realiza bajo circunstancia temporales y geográficas del contexto de estudio. Esta investigación es un estudio observacional con corte transversal, debido a que se aplicó el cuestionario de salud general de Goldberg GHQ-12 en una población específica en un momento determinado.

Muestra. La población de estudio que se consideró fueron los comerciantes de la Ciudad de Ambato, bajo los siguientes criterios de inclusión: que se encuentren registrados en el Servicio de Rentas Internas (SRI); a través de su Régimen Impositivo Simplificado (RISE); estén establecidos como una Pequeña y Mediana Empresa (PYME), acepten ser parte de la investigación a través de la firma del consentimiento informado; para el caso de este estudio la muestra es incidental, se contó con 280 personas que se dedican a la comercialización de diferentes productos; y relativamente sanos sin diagnóstico de enfermedades crónicas.

Instrumento. El GHQ -12 evalúa la dimensión general de la salud autopercibida por el paciente, pero además, permite distinguir dos subdimensiones: bienestar psicológico, en sus ítems 1, 2, 5, 7, 9 y 12, que es definido por Victoria y González (2014) como un constructo que “expresa el sentir positivo y el pensar constructivo del ser humano acerca de sí mismo, que se define por su naturaleza subjetiva vivencial y que se relaciona estrechamente con aspectos particulares del funcionamiento físico, psíquico y social” (p. 588). La segunda subdimensión es denominada por los autores como funcionamiento social y afrontamiento, evaluada en los ítems 3, 4, 6, 8, 10 y 11, y hace referencia a "la autoevaluación sobre la propia percepción de capacidad para atender los asuntos personales, afrontar las preocupaciones, decidir y atender los asuntos de la vida". Los ítems 3, 4, 5, 9 y 12 evalúan estado de ánimo, referido a cómo la persona se ha sentido en varias situaciones y el ítem 7 refleja la capacidad para disfrutar diversas actividades. Este cuestiona-rio también evalúa algunas funciones psicológicas y fisiológicas, tales como los ítems 1 y 2; la auto valoración del establecimiento y alcance de sus metas y propósitos de vida (ítems 10 y 11); y cómo enfrentan sus dificultades (ítems 6 y 8).

Son recomendados dos métodos para puntuar el GHQ-12: el dicotómico (0-0-1-1) (llamado el score GHQ) o una escala tipo Likert (0-1-2-3), puntuando cada ítem sobre una escala de 4 puntos (muy habitual, habitual, poco habitual y muy poco habitual). Hay pruebas que sugieren que un puntaje ordinal tipo Likert permite una mejor discriminación entre modelos competentes de análisis factoriales confirmatorios del GHQ-12 (Solís, Meda, \& Moreno, 2016).

Una escala tipo Likert fue usada en este estudio para producir una distribución más aceptable de los resultados del análisis paramétrico (con menos sesgo y curtosis). De acuerdo 
a este método el valor máximo obtenible es de 36 puntos y un mínimo de 0 puntos. El puntaje total se obtiene por la suma de los puntajes obtenidos en cada uno de los ítems. Puntajes elevados indican un estatus de salud más pobre. El GHQ-12 presentó buena confiabilidad en varios estudios conducidos por su autor con un coeficiente Alfa de Cronbach de 0,82 a 0,86 .

Procedimiento de recolección y Análisis de información. La recolección de información se realizó partiendo de brindar información a los participantes y concientizando en la importancia de esta investigación, posterior a este proceso se firma el consentimiento informado y se realiza la aplicación del cuestionario de salud general de Goldberg GHQ-12. Para el análisis de la información obtenida se crea una base de datos en SPSS, con los respectivos datos de las variables de estudio bienestar psicológico y estrés laboral, se registra los componentes del instrumento con sus respectivas valoraciones; mientras que, como variables de análisis se consideró el género y tipo de comercio que realizan. El análisis se realizó en base a estadística descriptiva e inferencial, con intervalos de confianza (IC) del 95\%; dentro de la primera se determina valores de media y desviación estándar, mientras que en el segundo se valida el nivel de estrés y de bienestar psicológico a través de análisis factorial simple del GHQ, con normalización Kaiser.

Aspectos Éticos. Para el manejo de la información de los participantes de la misma se estableció un consentimiento informado, el mismo que fue socializado a la población de estudio, explicando detalladamente: objetivos del estudio, responsabilidades asignadas en la misma, procedimientos que se aplicarán y duración que le llevará participar en este estudio; otro elemento importante que se explica a los involucrados en la investigación es si esta presenta algún tipo de riesgos para ellos, que para este caso no existe riesgo alguno; además, se describe los beneficios que recibirán al participar en la investigación, que para el caso es brindarles estrategias de afrontamiento del estrés; un elemento fundamental es la manera es que se manejará la confidencialidad de la información, que su participación es libre y voluntaria, que podrán retirarse en el momento que consideren pertinente y quienes son los responsables de la misma y con lo que deberá interactuar de manera permanente.

\section{RESULTADOS}

Análisis de validez - Estructura factorial confirmatoria: Se realizó un análisis factorial confirmatorio para dos factores. Se encontró que esta estructura factorial explica el $51.768 \%$ de la varianza. No se descartó ningún ítem, pues todos tenían una carga factorial superior a 
0.50. Para el factor 1 se agruparon los ítems 1, 3, 4, 7, 8, 12 y se nombró como "bienestar psicológico"; en el factor 2 se agruparon los ítems 2, 5, 6, 9, 10 y se nombró como "malestar psicológico o estrés laboral", se determinó el peso de cada uno de los componentes a partir del análisis factorial consignado en la tabla 3, los factores necesarios para obtener una solución factorial simple del GHQ, con normalización Kaiser fueron aceptadas. Como se esperaba, los factores corresponden a los más reportados tradicionalmente; el primero agrupa a los ítems positivos y, el segundo, a los ítems negativos. El total de varianza explicada por estos factores fue de $55.8 \%$.

\section{Tabla 1}

\section{Análisis de validez}

\begin{tabular}{|c|c|c|}
\hline \multirow{2}{*}{ GHQ - 12 ÍTEMS } & \multicolumn{2}{|c|}{ Componente } \\
\hline & 1 & 2 \\
\hline 1. ¿Ha podido concentrarse bien en lo que hace? & 0.6 & \\
\hline 2. ¿Sus preocupaciones le han hecho perder mucho sueño? & & 0.7 \\
\hline 3. ¿ Ha sentido que está jugando un papel útil en la vida? & 0.7 & \\
\hline 4. ¿Se ha sentido capaz de tomar decisiones? & 0.7 & \\
\hline 5. ¿Se ha sentido constantemente agobiado y en tensión? & & 0.8 \\
\hline 6. ¿Ha sentido que no puede superar sus dificultades? & & 0.7 \\
\hline 7. ¿Ha sido capaz de disfrutas sus actividades normales de cada día? & 0.7 & \\
\hline 8. ¿Ha sido capaz de hacer frentes a sus problemas? & 0.8 & \\
\hline 9. ¿Se ha sentido poco feliz y deprimido? & & 0.8 \\
\hline 10. ¿Ha perdido confianza en sí mismo? & & 0.6 \\
\hline 11. ¿Ha pensado que usted es una persona que no vale para nada? & & \\
\hline 12. ¿Se siente razonablemente feliz considerando todas las circunstancias? & 0.8 & \\
\hline Media & 0.7 & 0.7 \\
\hline Desviación estándar & 0.1 & 0.1 \\
\hline
\end{tabular}

Fuente: Resultados del cuestionario

Elaborado por: Taruchain, Freire y Mayorga, 2020

Se realizó después un análisis de consistencia interna mediante alfa de Cronbach y se encontró que ambos factores tienen índices adecuados de consistencia interna (.82 y .84, respectivamente), teniendo como resultado un Alfa de Cronbach promedio de 0.84, lo cual es aceptable, como se muestra en la tabla 2: 


\section{Tabla 2}

\section{Prueba de Alfa de Cronbach}

\section{GHQ - 12 ÍTEMS}

1. ¿Ha podido concentrarse bien en lo que hace?

2. ¿Sus preocupaciones le han hecho perder mucho sueño?

3. ¿ Ha sentido que está jugando un papel útil en la vida?

4. ¿Se ha sentido capaz de tomar decisiones?

5. ¿Se ha sentido constantemente agobiado y en tensión?

6. ¿Ha sentido que no puede superar sus dificultades?

7. ¿Ha sido capaz de disfrutas sus actividades normales de cada día?

8. ¿Ha sido capaz de hacer frentes a sus problemas?

9. ¿Se ha sentido poco feliz y deprimido?

10. ¿Ha perdido confianza en sí mismo?

11. ¿Ha pensado que usted es una persona que no vale para nada?

12. ¿Se siente razonablemente feliz considerando todas las circunstancias?

El alfa de Cronbach

Fuente: Resultados del cuestionario

Elaborado por: Taruchain, Freire y Mayorga, 2020

Se establece un análisis de variables sociodemográficas, en él se estable que existe mayor población femenina que masculina; dentro de edades comprendidas en la del grupo poblacional del adulto medio, prevalece nivel de escolaridad primaria; y el estado civil casado y unión libre, como se muestra en la tabla 3 :

\section{Tabla 3}

\section{Variables Sociodemográficas}

\begin{tabular}{clrr}
\hline Variables Sociodemográficas & Frecuencia & Porcentaje \\
\hline \multirow{3}{*}{ Edad } & Adulto Joven & 93 & $33 \%$ \\
& Adulto Medio & 142 & $51 \%$ \\
& Adulto Mayor & 45 & $16 \%$ \\
Sexo & Hombre & 111 & $40 \%$ \\
& Mujer & 169 & $60 \%$ \\
\multirow{5}{*}{ Estrato } & Alto & 36 & $13 \%$ \\
& Medio & 163 & $58 \%$ \\
& Bajo & 81 & $29 \%$ \\
\hline
\end{tabular}


Tabla 4 (continuación)

Variables Sociodemográficas

\begin{tabular}{clrr}
\hline Variables Sociodemográficas & Frecuencia & Porcentaje \\
\hline \multirow{4}{*}{ Escolaridad } & Primaria & 149 & $53 \%$ \\
& Secundaria & 78 & $28 \%$ \\
& Técnico & 53 & $19 \%$ \\
& Profesional & 0 & $0 \%$ \\
& Soltero & 77 & $28 \%$ \\
Estado & Casado/unión libre & 125 & $45 \%$ \\
Civil & Separado/divorciado & 61 & $22 \%$ \\
& Viudo & 17 & $6 \%$
\end{tabular}

Fuente: Resultados del cuestionario

Elaborado por: Taruchain, Freire y Mayorga, 2020

En la tabla 4 se evidencia que la población de comerciantes no profesionales tiene un nivel de estrés laboral entre muy alto y alto en un $61 \%$, lo que refleja que la mayor parte presente un riesgo elevado de desarrollar enfermedades:

\section{Tabla 5}

Niveles de estrés percibidos

\begin{tabular}{lcc}
\hline Niveles de estrés percibidos & N & $\%$ \\
\hline Muy Alto & 91.00 & $33 \%$ \\
Alto & 77.00 & $28 \%$ \\
Medio & 48.00 & $17 \%$ \\
Bajo & 39.00 & $14 \%$ \\
Muy Bajo & 25.00 & $9 \%$ \\
\hline Total & $\mathbf{2 8 0 . 0 0}$ & $\mathbf{1 0 0} \%$
\end{tabular}

Fuente: Resultados del cuestionario

Elaborado por: Taruchain, Freire y Mayorga, 2020

En la tabla 5 se evidencia que la población de comerciantes no profesionales dentro de lo que es el bienestar psicológico auto percibido, la mayor parte lo valora como muy bajo y bajo en un 59\%, lo que conlleva a su desempeño emocional y de autoestima indeseable: 


\section{Tabla 6}

Bienestar Psicológico

\begin{tabular}{|c|c|c|}
\hline Bienestar Psicológico & $\mathbf{N}$ & $\%$ \\
\hline Muy Alto & 23.00 & $8 \%$ \\
\hline Alto & 31.00 & $11 \%$ \\
\hline Medio & 61.00 & $22 \%$ \\
\hline Bajo & 88.00 & $31 \%$ \\
\hline Muy Bajo & 77.00 & $28 \%$ \\
\hline Muy Alto & 23.00 & $8 \%$ \\
\hline Total & 280.00 & $100 \%$ \\
\hline
\end{tabular}

\section{CONCLUSIONES}

Se concluye que, en la muestra de trabajadores de centros de comercialización de la ciudad de Ambato, existe la presencia de síntomas de una etapa grave de estrés laboral. Lo cual refiere que, si bien las puntuaciones se encuentran estadísticamente significativas, a nivel clínico existe un malestar grave que prevalece en la población de estudio que, sin la intervención necesaria, podría escalar en un Síndrome de Burnout.

El resultado principal permite demostrar que este test es válido no solo para profesionales de la salud, sino que se puede aplicar en otro tipo de personas pues estás también sufren de estrés el cual afecta directamente a su bienestar emocional; pues son dimensiones que son parte del ser humano. Los resultados son relevantes partiendo de la premisa que el estrés laboral es un problema de salud pública que, si no es detectado a tiempo y de maneras oportuna desencadena en problemas de salud físicos, psicológicos y emocionales de cada individuo, tomando en cuenta que toda enfermedad psicofisiológica impide que los seres humanos realicen efectivamente sus funciones.

De ahí que se puede determinar que el estrés altera el mecanismo tanto físico como psicológico, de todas las personas indistintamente de la actividad que realicen y que por ende debe ser afrontada con la seriedad del caso porque si no se tiene en cuenta puede ir agravando la situación hasta llegar a desencadenar enfermedades realmente graves, ya que afecta la mente, produce alteraciones en la personalidad y puede ser reflejando en afecciones corporales. Desde el momento que se ve al estrés como algo que existe, se puede decir que tanto un individuo al igual que grupo de personas, se pueden ver afectados por distintos tipos de crisis que puedan crearles situaciones de estrés en sus distintos ámbitos de su vida tales como en los físicos y 
como en los psicológicos. Es por eso que al estrés se le define como las respuestas no específicas ante situaciones donde las personas puedan verse vulnerables y sobrecargar su resistencia.

\section{REFERENCIAS BIBLIOGRAFICAS}

Arón, A. (2017). Violencia en la familia. Programa de intervención en red: la experiencia de San Bernardo. Santiago, Chile: Editorial Galdoc.

Ávila, J. (11 de Septiembre de 2014). El estrés es un problema de salud del mundo actual. Recuperado el 11 de Marzo de 2019, de http://www.revistasbolivianas.org.bo/scielo.php?pid=S231002652014000100013\&script=sci_arttext

Brabete, A. (2014). El Cuestionario de Salud General de 12 items (GHQ-12): estudio de traducción y adaptación de la versión rumana. Revista Iberoamericana de Diagnóstico y Evaluación, 1(37), 11-29. Obtenido de https://www.redalyc.org/pdf/4596/459645433002.pdf

De la Cruz, E. (2017). Estrés laborañ síndrome de Burnout en los profesionales de enfermería de atención hospitalaria. Trabajo de fin de grado. España: Universidad de Valladolid. Recuperado el 8 de diciembre de 2019, de https://uvadoc.uva.es/bitstream/handle/10324/5629/TFGH123.pdf;jsessionid=19BB9D23E113CC85FE87647E696D7840?sequence=1

Díaz, F., \& Gómez, I. (2016). la investigación sobre el síndrome de Burnout en

Latinoamérica. (U. d. Norte, Ed.) Revista de Psicología desde El Caribe, 33(1), 112131. Recuperado el 7 de diciembre de 2019, de http://www.scielo.org.co/pdf/psdc/v33n1/v33n1a09.pdf

Gil-Monte, P. (2016). El síndrome de quemarse por el trabajo (burnout). Valencia: Diputación de Valencia.

Gil-Monte, P., Salanova, M., Aragón, J., \& Schaufeli , W. (2016). El Síndrome de quemarse por el trabajo en Servicios Sociales. Valencia: Diputación de Valencia.

Ochoa, A., \& Pacheco, K. (Marzo de 2019). Síndrome de burnout: Validez del Maslach Burnout Invetory - General Survey en una población ecuatoriana. Propuesta de artículo para la obtención del título de Maestría en Seguridad y Salud Ocupacional. 
Ecuador: Universidad Espíritu Santo. Recuperado el 11 de diciembre de 2019, de http://repositorio.uees.edu.ec/bitstream/123456789/3031/1/OCHOA\%20DE\%20LA\% 20VERA\%20Y\%20PACHECO\%20MONTECE.pdf

Solís, P., Meda, R., \& Moreno, B. (2016). Estructura factorial del Cuestionario de Salud General GHQ-12 en población general de México. Revista Salud y Sociedad, 7(1), 6276. Obtenido de https://www.researchgate.net/publication/304626925_Estructura_factorial_del_Cuesti onario_de_Salud_General_GHQ-12_en_poblacion_general_de_Mexico

Velázquez, j., \& Saldívar, A. (2018). Síndrome de burnout y estrés laboral: Una revisión. Revista de Medicina familiar, 10(2), 65-72.

Victorio, C., \& González, I. (2014). La categoría Bienestar psicológico. Su relación con otras categorías sociales. Revista Cubana de Medicina GeneralIntegral, 6(16), 586-892.

Villa, I. (2016). Propiedades psicométricas del Cuestionario de Salud General de Goldberg GHQ-12 en una institución hospitalaria de la ciudad de Medellín. Avances en Psicología Latinoamericana, 31(3), 532-545. Obtenido de http://www.scielo.org.co/pdf/apl/v31n3/v31n3a07.pdf

Yegler, M., Diez, T., Gómez, J., \& Miralles, T. (2013). Determinación del bienestar psicológico de un equipo de enfermería de un servicio de urgencia hospitalaria aplicando e cuestionario de salud general de Goldberg. Revista Sociedad Española de Enfermería de Urgencias y Emergencias(16). Obtenido de http://www.enfermeriadeurgencias.com/ciber/PRIMERA_EPOCA/2003/octubre/psico logico.htm

\section{Ciencia}




\section{PARA CITAR EL ARTÍCULO INDEXADO}

Taruchaín Pozo, L. F., Freire Palacios, V. A., \& Mayorga Álvarez, M. de los Ángeles. (2020). Bienestar psicológico y estrés laboral en trabajadores no profesionales de la ciudad de Ambato, aplicando el cuestionario de salud general de Goldberg GHQ-12. Ciencia Digital, 4(1.1.), 3244. https://doi.org/10.33262/cienciadigital.v4i1.1.1162

\section{Ciencia}

El artículo que se publica es de exclusiva responsabilidad de los autores y no necesariamente reflejan el pensamiento de la Revista Ciencia Digital.

El artículo queda en propiedad de la revista y, por tanto, su publicación parcial y/o total en otro medio tiene que ser autorizado por el director de la Revista Ciencia Digital.
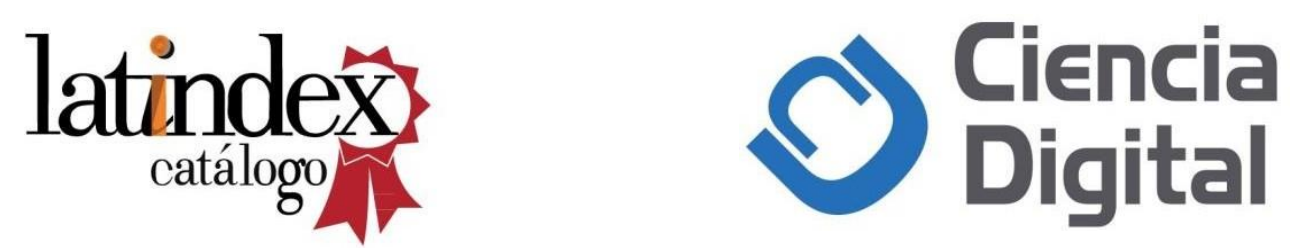